\title{
Ringholes and closed timelike curves
}

\author{
Pedro F. González-Díaz \\ Centro de Física "Miguel Catalán", Instituto de Matemáticas y Física Fundamental, \\ Consejo Superior de Investigaciones Científicas, Serrano 121, 28006 Madrid (SPAIN)
}

(March 1, 1996)

\begin{abstract}
It is shown that in a classical spacetime with multiply connected space slices having the topology of a torus, closed timelikes curves are also formed. We call these spacetimes ringholes. Two regions on the torus surface can be distinguished which are separated by angular horizons. On one of such regions (that which surrounds the maximum circunference of the torus) everything happens like in spherical wormholes, but the other region (the rest of the torus surface), while still possessing a chronology horizon and non-chronal region, behaves like a converging, rather than diverging, lens and corresponds to an energy density which is always positive for large speeds at or near the throat. It is speculated that a ringhole could be converted into a time machine to perform time travels by an observer who would never encounter any matter that violates the classical averaged weak energy condition. Based on a calculation of vacuum fluctuations, it is also seen that the angular horizons can prevent the emergence of quantum instabilities near the throat.
\end{abstract}

PACS number(s): 04.20.Cv, 04.62.+v

\section{INTRODUCTION}

Time travels have become a scientific possibility in recent years [1]. Spacetimes possessing closed timelike curves (CTCs) were originally discussed by Gdel [2], but it only was after recognition that negative-energy states can occur in semiclassical general relativity that time travels have been considered in greater detail. Recently, the subject of CTC was revived in two apparently quite different contexts. The first involves tunneling through wormholes in four-dimensional Einstein gravity [3]. The second, which we shall not discuss here, concerns solutions to gravity with infinite parallel cosmic strings which move with high velocity relative to each other [4]. Although the latter does not entail negative energies, the moving straight strings can only produce CTCs if they are infinite in length [5] and could therefore not exist in a finite universe.

Time travels through four-dimensional wormholes entail an inward pressure on the tunnel so great that it ultimately meant a matter with negative energy density [3]. Although negative energy is becoming commonplace in quantum and semiclassical gravity [6], it would imply violation of the averaged weak energy condition in classical general relativity. This situation is deeply troublesome and, if one calculate quantum fluctuations of vacuum in wormholes where one can provide them with a more natural context for negative energies to occur, then divergences of the stress-energy tensor arise [7] that may make the problem even more acute [5]. It appears therefore that for a classical solution to Einstein equations with CTCs to offer an acceptable scenario for time travels, such a solution must provide with finite geometrical objects able to live in our universe and simultaneously entail classical stress-energy tensors with positive energy density.
The ultimate reason for traversible wormholes to have negative energy densities is that their very geometry necessarily requires any bundle of rays entering one wormhole's mouth radially converging, to emerge from the other mouth radially diverging [8]. This diverging-lens action, on the other hand, prevents the spacetime structure from pinching off at the throat and, moreover, is responsible for the existence of CTCs in it. However, though the diverging-lens effect protects the wormhole against the classical instability produced by the wave's pileup at or near the throat, it is unable to prevent the wormhole from quantum instabilities originated by electromagnetic vacuum fluctuations $[7,8]$. This situation has led Hawking to suggest his chronology protection conjecture, according to which the laws of physics do not allow the appearance of CTCs [5].

A possible way out from this situation could be making recourse to tunnels with topologies other than spherical. The most obvious candidate for one such topologies is the torus. One would then tunnel from a region of Euclidean space by means of a torus whose transverse crosssectional area would shrink down to a minimum size at the throat, increasing thereafter to finally be embedded again in another region of Euclidean space (Fig. 2). We shall denote this geometric construct a ringhole. The interesting thing one would expect about these ringholes is that, although bundles of rays originally converging in the direction tangent to the torus at points on the maximum circunference will still emerge diverging in the same direction, bundles of rays originally diverging in the direction tangent to the torus at points on the minimum circunference would now emerge converging in the same direction at the other ringhole's mouth. Then, if the surface surrounding the maximum circunference is able to prevent the whole geometrical construct from pinching off at the throat, by traversing the ringhole along a 
world line which would pass through the neighborhood of the minimum circunference, one might expect the appearance of CTCs without any violation of the averaged weak energy condition [9], whenever one ringhole's mouth is kept at rest and the other moves at sufficiently high velocity.

In this paper we shall explore this possibility by constructing the metric of a traversible ringhole which is solution to the Einstein equations, and converting it into a time machine. It is seen that also in this case, on the neighborhood of the maximum circunference of the torus only the leftward chronology (Cauchy) horizon and nonchronal region with CTCs survive, while the averaged weak energy condition is violated at or near the throat. However, on the neighborhood of the minimum circunference of the torus, what survive are the rightward chronology (Cauchy) horizon and non-chronal region with CTCs, while, quite remarkably, the averaged weak energy condition holds at or near the throat. Thus, we find no classical obstruction for an advanced civilization to construct a time machine with toroidal topology by using traversible ringholes with just one of their mouths kept moving with respect to the other mouth, whenever the travel itinerary passes through the throat along a world line near the minimum toroidal circunference. By using the method of Kim and Throne [7], it has been also shown that, in this case, vacuum fluctuations do not destabilize the accelerating ringhole either.

The body of this paper is organized as follows: In Sec. II we review the way in which the static metric describing the spacetime generated by a distribution of matter with the topology of a torus can be obtained, and discuss the existence of apparent angular horizons of this metric. In Sec. III we consider the existence of what we call ringholes, that is tunnels in Lorentzian spacetime with toroidal symmetry, deriving their metric so as the characteristics of the stress-energy tensor that make these ringholes compatible with general relativity. Lensing actions in the ringhole space are also considered. Then in Sec. IV we convert ringhole into time machine and analyse the causal and non-causal structure of the resulting space in relation with the possibility of using accelerating ringholes to travel back- and forward in time, and in Sec. V we compute the quantum effect of vacuum polarization following the procedure of Kim and Thorne in [7]. Finally, we summarize and briefly comment on our results in Sec. VI.

\section{STATIC METRIC ON THE TORUS}

Let us consider the gravitational field created by a distribution of matter having the symmetry of a torus. We shall obtain the static space-time metric corresponding to any constant $2+1$ surface with such a symmetry.

Defining the Cartesian coordinates as in Fig.1, it follows $x=m \sin \varphi_{1}, y=m \cos \varphi_{1}, z=b \sin \varphi_{2}$, so that

$$
d x^{2}+d y^{2}+d z^{2}=m^{2} d \varphi_{1}^{2}+b^{2} d \varphi_{2}^{2},
$$

with

$$
\begin{aligned}
& m=a-b \cos \varphi_{2} \\
& n=b-a \cos \varphi_{2},
\end{aligned}
$$

$a$ and $b$ being the radius of the circunference generated by the circular axis of the torus and that of a torus section, respectively.

We can assume then for the static metric corresponding to a distribution of matter with the symmetry of a torus the general expression

$$
d s^{2}=-e^{\Phi} d t^{2}+e^{\Psi} d r^{2}+m^{2} d \varphi_{1}^{2}+b^{2} d \varphi_{2}^{2},
$$

where

$$
r=\left(a^{2}+b^{2}-2 a b \cos \varphi_{2}\right)^{\frac{1}{2}},
$$

and $\Phi$ and $\Psi$ will generally depend on $r$ and $t$.

Denoting by $x^{0}, x^{1}, x^{2}, x^{3}$, respectively, the coordinates on the torus $c t, r, \varphi_{1}$ and $\varphi_{2}$, the nonzero components of the metric tensor take the general form $g_{00}=-e^{\Phi}$, $g_{11}=e^{\Psi}, g_{22}=m^{2}$ and $g_{33}=b^{2}$. Using then

$$
\begin{gathered}
\frac{d m}{d r}=\frac{r}{a}, \frac{d m}{d \varphi_{2}}=b \sin \varphi_{2}, \quad \frac{d n}{d r}=\frac{r}{b}, \frac{d n}{d \varphi_{2}}=a \sin \varphi_{2} \\
\frac{d a}{d r}=\frac{r}{m}, \quad \frac{d b}{d r}=\frac{r}{n},
\end{gathered}
$$

one can calculate the components of the affine connection and hence those of the Ricci curvature tensor. Finally, one has for the Einstein equations corresponding to the gravitational field of a matter distribution with the symmetry of a torus

$$
\begin{aligned}
& e^{-\Psi}\left[r^{2}\left(\frac{1}{m^{2} a^{2}}+\frac{1}{n^{2} b^{2}}\right)+\left(\frac{1}{m a}+\frac{1}{n b}\right)\left(\frac{1}{2} r \Psi^{\prime}-1\right)\right. \\
& \left.-\frac{r^{2}}{m n a b}\right]-\frac{\cos \varphi_{2}}{m b}=\frac{8 \pi G}{c^{4}} T_{0}^{0}
\end{aligned}
$$

$$
\begin{gathered}
-e^{-\Psi}\left[\frac{1}{2}\left(\frac{1}{m a}+\frac{1}{n b}\right) \Phi^{\prime} r+\frac{r^{2}}{m n a b}\right] \\
-\frac{\cos \varphi_{2}}{m b}=\frac{8 \pi G}{c^{4}} T_{1}^{1}
\end{gathered}
$$




$$
\begin{gathered}
e^{-\Psi}\left[\frac{r^{2}}{n^{2} b^{2}}-\frac{r\left(\Phi^{\prime}-\Psi^{\prime}\right)}{2 n b}-\frac{1}{n b}\right] \\
+F\left(r, \varphi_{2}, \Psi, \Phi\right)=\frac{16 \pi G}{c^{4}} T_{2}^{2} \\
e^{-\Psi}\left[\frac{r^{2}}{m^{2} a^{2}}-\frac{r\left(\Phi^{\prime}-\Psi^{\prime}\right)}{2 m a}-\frac{1}{m a}\right] \\
+F\left(r, \varphi_{2}, \Psi, \Phi\right)=\frac{16 \pi G}{c^{4}} T_{3}^{3} \\
-\frac{1}{2} r \dot{\Psi}\left(\frac{1}{m a}+\frac{1}{n b}\right) e^{-\Psi}=\frac{8 \pi G}{c^{4}} T_{0}^{1}, \\
\text { where the prime denotes differentiation with respect to } \\
r, \text { the overhead dot accounts for time derivative, and } \\
F\left(r, \varphi_{2}, \Psi, \Phi\right)=-\frac{1}{2} e^{-\Psi}\left(\Phi^{\prime \prime}-\frac{1}{2} \Phi^{\prime} \Psi^{\prime}+\frac{1}{2} \Phi^{\prime 2}\right) \\
+\frac{1}{2} e^{-\Phi}\left(\ddot{\Psi}-\frac{1}{2} \dot{\Psi} \dot{\Phi}+\frac{1}{2} \dot{\Psi}^{2}\right) .
\end{gathered}
$$

General solutions to these equations look quite complicate. Nevertheless, we shall derive a solution for the vacuum case whenever $a$ and $b$ are constant.

Outside the space region occupied by the matter which creates the gravitational field we must have $T_{i}^{i}=0, i=$ $0,1, \ldots 3$. For this vacuum case, using $r^{2}=m a+n b$, one can obtain from the first pair of Einstein equations

$$
\frac{1}{2} r\left(\Phi^{\prime}+\Psi^{\prime}\right)=1-\left(\frac{m a}{n b}+\frac{n b}{m a}\right) .
$$

Likewise, from the second pair of Einstein equations in the vacuum we arrive at

$$
\frac{1}{2} r\left(\Phi^{\prime}-\Psi^{\prime}\right)=1+\left(\frac{m a}{n b}+\frac{n b}{m a}\right) .
$$

Clearly, in the vacuum we also have from (2.12)

$$
\dot{\Psi}=0 .
$$

If we assume then a torus surface where the radii $a$ and $b$ are both constant (which we shall denote a constant torus), the solutions to (2.14) and (2.15) become

$$
\Phi=\ln \frac{r^{2}}{C_{2}}
$$

$$
\Psi=\ln \left[\frac{C_{1}}{r^{4}\left(1-\frac{A^{2}}{r^{4}}\right)^{2}}\right],
$$

where $A=a^{2}-b^{2}$ and $C_{1}$ and $C_{2}$ are integration constants which would be given in terms of the mass distribution with the symmetry of a torus. The expression of these constants will not be of any importance in the discussion to follow.

Thus, the following three-dimensional metric is finally obtained for a torus symmetry with constant radii $a$ and $b$ :

$$
\begin{gathered}
d s^{2}=-C_{2} r^{2} d t^{2}+ \\
{\left[\frac{C_{1}}{r^{4}\left(1-\frac{A^{2}}{r^{4}}\right)^{2}}+\frac{4 b^{2}}{2 B-r^{2}\left(1+\frac{A^{2}}{r^{4}}\right)}\right] d r^{2}+m^{2} d \varphi_{1}^{2}} \\
=-C_{2} r^{2} d t^{2}+b^{2}\left[1+\frac{C_{1} a^{2} \sin ^{2} \varphi_{2}}{r^{6}\left(1-\frac{A^{2}}{r^{4}}\right)^{2}}\right] d \varphi_{2}^{2}+m^{2} d \varphi_{1}^{2},
\end{gathered}
$$

where $B=a^{2}+b^{2}$, with $a$ and $b$ constant and $a>b$. Metric (2.19) is defined for $0 \leq t \leq \infty, a-b \leq r \leq$ $a+b, 0 \leq \varphi_{1}, \varphi_{2} \leq 2 \pi$, and describes the space-time geometry on a torus generated by varying angles $\varphi_{1}$ and $\varphi_{2}$ only; i.e.: the space-time geometry on a torus with constant radii $a$ and $b$ generated by moving about its entire surface.

Of most interest is the variation of the metric with angle $\varphi_{2}$. On $\varphi_{2}=\pi$, where $r=r_{\max }=a+b$ (the radius of the maximum circunference of the torus) the metric becomes singular. As $\varphi_{2}$ decreases from $\pi$ to $\varphi_{2}^{c}=\arccos \frac{b}{a}$, $r$ decreases from $r_{\max }$ to $r=\sqrt{A}$ where the metric becomes singular as well. The metric is again singular on $\varphi_{2}=0$ where $r=r_{\min }=a-b$ (the radius of the minimum circunference of the torus). Of course, the same singularity as that on $\varphi_{2}^{c}$ will also occur on $2 \pi-\varphi_{2}^{c}$. One would then cut the points on the two extremum circunferences of the torus and on the two circunferences for which $r=\sqrt{A}$ out of the manifold defined by the coordinates $t, r, \varphi_{1}$ (or $\left.t, \varphi_{1}, \varphi_{2}\right)$, and so divide this manifold into four disconnected components.

However, one would expect the singularities on $r=$ $r_{\min }$ and $r=r_{\max }$ to play an analogous role to that of the trivial singularities of polar coordinates when $\theta=0$ and $\theta=\pi$. Moreover, calculation of the curvature invariants, such as $R^{i j k l} R_{i j k l}$, shows that all of these invariants are finite on the above points where the metric becomes singular in coordinates $t, r, \varphi_{1}$ (or $\left.t, \varphi_{1}, \varphi_{2}\right)$. This strongly suggests that all of these singularities are not true singularities but only arise from the choice of coordinates [9]. In fact, if we first introduce the new real coordinates

$$
u=t+\frac{C_{3}}{4 A} \ln \left(\frac{A-r^{2}}{A+r^{2}}\right), \quad v=t-\frac{C_{3}}{4 A} \ln \left(\frac{A-r^{2}}{A+r^{2}}\right),
$$


with $C_{3}=\sqrt{\frac{C_{1}}{C_{2}}}$, the metric takes the form

$$
d s^{2}=-C_{2} r^{2} d u d v+\frac{4 b^{2} d r^{2}}{2 B-r^{2}\left(1+\frac{A^{2}}{r^{4}}\right)}+m^{2} d \varphi_{1}^{2},
$$

where

$$
r^{2}=A \tanh \left[\frac{A(u-v)}{C_{3}}\right],
$$

which still is singular on $r=r_{\max }$ and $r=r_{\min }$. Introducing then the additional complex coordinate

$$
w=i \varphi_{1}+\frac{b}{2 \sqrt{A}} \arcsin \left[\frac{a\left(r^{2}-A\right)}{b\left(r^{2}+A\right)}\right],
$$

allows one to re-write metric (2.19) into the final form in coordinates $u, v, w$

$$
d s^{2}=-C_{2} r^{2} d u d v+m^{2}|d w|^{2},
$$

where

$$
\begin{gathered}
i \varphi_{1}=\frac{1}{2}\left(w-w^{*}\right) \\
r^{2}=A\left(\frac{1+\sin \varphi_{2}^{c} \sin x}{1-\sin \varphi_{2}^{c} \sin x}\right),
\end{gathered}
$$

in which $x=\frac{\sqrt{A}}{b}\left(w+w^{*}\right)$ is real and * denotes complex conjugation.

It is easy to see that metric (2.23) is real and in fact regular everywhere in the complete three-manifold for which $r_{\min } \leq r \leq r_{\max }$. We note finally that by rotating $\varphi_{1} \rightarrow \tilde{\varphi}_{1}=-i \varphi_{1}$, coordinate $w$ unfolds into two distinct real coordinates $w \rightarrow w_{1}$ and $w^{*} \rightarrow w_{2}$, and hence metric (2.23) becomes

$$
d s^{2} \rightarrow d \tilde{s}^{2}=d s^{2}-m^{2}\left(|d w|^{2}-d w_{1} d w_{2}\right) .
$$

\section{RINGHOLE SPACETIME}

One would expect that creation of traversible ringholes respecting Einstein equations in such a way that classical general relativity be valid everywhere, must be accompanied by CTCs in some non-chronal spacetime sectors that would appear at late time, and by violation of the averaged weak energy condition only on some restricted, classically forbidden spatial regions.

A static ringhole having toroidal topology would be traversible if a two-torus surrounding one of its mouths where spacetime is nearly flat can be regarded as an outer trapped surface to an observer looking through the ringhole from the other mouth [3]. The static spacetime metric for one such single, traversible ringholes can be written in the form $d s^{2}=-d t^{2}+\left(\frac{n_{l}}{r_{l}}\right)^{2} d l^{2}+m_{l}^{2} d \varphi_{1}^{2}+\left(l^{2}+b_{0}^{2}\right) d \varphi_{2}^{2}$,

where $-\infty<t<+\infty,-\infty<l<+\infty, \varphi_{1}$ and $\varphi_{2}$ are as given for the torus metric (2.19), $l$ is the proper radial distance of each transversal section of the torus, and

$$
\begin{gathered}
m_{l}=a-\left(l^{2}+b_{0}^{2}\right)^{\frac{1}{2}} \cos \varphi_{2} \\
n_{l}=\left(l^{2}+b_{0}^{2}\right)^{\frac{1}{2}}-a \cos \varphi_{2} \\
r_{l}=\left[a^{2}+l^{2}+b_{0}^{2}-2\left(l^{2}+b_{0}^{2}\right)^{\frac{1}{2}} a \cos \varphi_{2}\right]^{\frac{1}{2}}
\end{gathered}
$$

Metric (3.1) is of special interest as it gives us a particularly simple example of a traversible ringhole which is readily generalizable. One can convert (3.1) into the more general static ringhole metric (note that $r d r=n d b$ )

$$
\begin{aligned}
& d s^{2}=-d t^{2} e^{2 \Phi}+\frac{d r^{2}}{1-\frac{K(b)}{b}}+m^{2} d \varphi_{1}^{2}+b^{2} d \varphi_{2}^{2} \\
& =-d t^{2} e^{2 \Phi}+\left(\frac{n}{r}\right)^{2} d l^{2}+m^{2} d \varphi_{1}^{2}+b^{2} d \varphi_{2}^{2}
\end{aligned}
$$

if we let $l= \pm\left(b^{2}-b_{0}^{2}\right)^{\frac{1}{2}}, \Phi=0$ and $K(b)=\frac{b_{0}^{2}}{b}$, where the minus sign applies on the left side of the throat and the plus sign does on the right side. $\Phi$ will generally be now given as a function of the ringhole mass and the geometric parameters $a$ and $b$. At the throat $l=0$, so that the throat radius becomes $b_{0}$.

Metric (3.5) can be regarded as a generalization to toroidal symmetry from the static, spherical wormhole metric [3]. The reduction transformations $a \rightarrow 0, \varphi_{2} \rightarrow$ $\theta+\frac{\pi}{2}, \varphi_{1} \rightarrow \phi$, where $\theta$ and $\phi$ are the angular polar coordinates on the two-sphere, leads to $r=n=b$, $m=r \sin \theta$, with which metric (3.5) reduces to

$$
d s^{2}=-d t^{2} e^{2 \Phi}+d l^{2}+r^{2}\left(d \theta^{2}+\sin ^{2} \theta d \phi^{2}\right),
$$

which, in fact, is the static metric used by Morris et al. [3] if we take $r \cong|l|-M \ln \left(\frac{|l|}{b_{0}}\right)$ and $\Phi \cong-\frac{M}{|l|}$ far from the throat, and $M$ is regarded to be the wormhole mass. One must add $\frac{\pi}{2}$ to $\theta$ to get $\varphi_{2}$ because of the different choice of the ringhole symmetry axis which is here taken to be the axis $z$ of Fig.1.

Metric (3.5) will be viewed to represent tunneling through a traversible ringhole, and satisfy Einstein equations for convenient stress-energy tensors for the following reasons:

(i) As $l$ increases from $-\infty$ to $0, b$ decreases monotonously from $+\infty$ to a minimum value $b_{0}$ (the throat radius), and as $l$ increases onward to $+\infty, b$ increases monotonously to $+\infty$ again. 
(ii) For (3.5) to describe a ringhole we must embed it in a three-dimensional Euclidean space at a fixed time $t[3]$. We should consider a three-geometry that would respect the symmetry of a torus and satisfy $a \geq b<l$, so it will suffice confining attention to the maximum- and minimum-circunference slices, i.e. $\varphi_{2}=\pi, 0$, through it. In the first case, $r=m=n=a+b$, and

$$
d s^{2}=\frac{d r^{2}}{1-\frac{b_{0}^{2}}{b^{2}}}+r^{2} d \varphi_{1}^{2}
$$

We wish now visualize the slice (3.7) as removed from spacetime (3.5) and embedded in three-dimensional Euclidean space. For the embedding space, one takes a space with cylindrical coordinates $z, r, \phi$,

$$
d s^{2}=d z^{2}+d r^{2}+r^{2} d \phi^{2} .
$$

The embedded surface will be axially symmetric and can therefore be described by a function $z=z(r)$. From (3.8) we have

$$
d s^{2}=\left[1+\left(\frac{d z}{d r}\right)^{2}\right] d r^{2}+r^{2} d \phi^{2}
$$

Metric (3.9) will be the same as metric (3.7) if we identify the coordinates $r, \phi$ of the embedding space with the coordinates $r, \varphi_{1}$ of the ringhole spacetime, and if we require the function $z(r)$ to satisfy

$$
\frac{d z}{d r}=\left(\frac{b^{2}}{b_{0}^{2}}-1\right)^{-\frac{1}{2}}
$$

This equation displays the way in which, provided we have a fixed $a \geq b$, the function $b \equiv b(l)$ shapes the ringhole spatial geometry. In Fig. 2 we picture the surface $z(r)$.

If we confine attention to the minimum-circunference slice, $\varphi_{2}=0$, through the geometry of three-space at a fixed time, then $r=m=-n=a-b$ and the metric (3.7) is obtained again. Thus, no matter the election of slice, we always achieve the same condition (3.10).

(iii) From the Einstein equations (2.8) and (2.9) we obtain for the metric components of (3.5) with $\Phi=0$ :

$$
\begin{gathered}
-\frac{b_{0}^{2}}{n b^{3}}\left(2+\frac{m a}{n b}+\frac{n b}{m a}\right)+ \\
\left(1-\frac{b_{0}^{2}}{b^{2}}\right)\left(\frac{m a}{n^{2} b^{2}}+\frac{n b}{m^{2} a^{2}}\right)=\frac{8 \pi G}{c^{4}}\left(T_{0}^{0}-T_{1}^{1}\right) .
\end{gathered}
$$

The stress-energy tensor components $T_{0}^{0}$ and $T_{1}^{1}$ in (3.11) cannot be directly given in terms of, respectively, an energy density, $\rho$, and a tension per unit area, $\sigma$, for the toroidal symmetry, because these tensor components should explicitly depend on $r$ (see Fig. 1) whereas $\rho$ and $\sigma$ must be defined in function of the normal to a surface element on the torus, along the $b$-direction. Since $\frac{d r}{d b}=\frac{n}{r}$, in the neighborhood of the ringhole throat $\left(b \simeq b_{0}\right)$, we have then

$$
\begin{gathered}
\rho c^{2}-\sigma=\left(\frac{n}{r}\right)^{3}\left(T_{0}^{0}-T_{1}^{1}\right) \simeq \\
-\frac{c^{4} n^{2} b_{0}^{2}}{8 \pi G r^{3} b^{3}}\left(2+\frac{m a}{n b}+\frac{n b}{m a}\right) .
\end{gathered}
$$

The requirement that ringholes be connectible to asymptotically flat spacetime entails at the throat that the embedding surface flares outward for $2 \pi-\varphi_{2}^{c}>\varphi_{2}>$ $\varphi_{2}^{c}$ and "flares inward" for $-\varphi_{2}^{c}<\varphi_{2}<\varphi_{2}^{c}$. One would expect these flarings to be mathematically expressed by the conditions

$$
\begin{gathered}
\frac{d^{2} r}{d z^{2}}>0 \text { for } 2 \pi-\varphi_{2}^{c}>\varphi_{2}>\varphi_{2}^{c} \\
\frac{d^{2} r}{d z^{2}}<0 \text { for }-\varphi_{2}^{c}>\varphi_{2}>\varphi_{2}^{c},
\end{gathered}
$$

at or near the throat.

From (3.10) we can obtain

$$
\frac{d^{2} r}{d z^{2}}=\frac{b r}{n b_{0}^{2}}
$$

which in fact satisfies both conditions (3.13) and (3.14). Now, since $r^{3} b^{3}$ and $b_{0}^{2}$ are both positive, from (3.12) we obtain

$$
\operatorname{sign}\left[\rho c^{2}-\sigma\right]=\operatorname{sign}\left[-\left(2+\frac{m a}{n b}+\frac{n b}{m a}\right)\right]
$$

at or near the ringhole throat. It is easy to check that, since $r$ and $m$ are always positive for any value of $\varphi_{2}$, and $n$ is positive for $2 \pi-\varphi_{2}^{c}>\varphi_{2}>\varphi_{2}^{c}$ and negative for $-\varphi_{2}^{c}<\varphi_{2}<\varphi_{2}^{c}$, we have finally

$$
\begin{gathered}
\rho c^{2}-\sigma<0 \text { for } 2 \pi-\varphi_{2}^{c}>\varphi_{2}>\varphi_{2}^{c} \\
\rho c^{2}-\sigma>0 \text { for }-\varphi_{2}^{c}<\varphi_{2}<\varphi_{2}^{c}
\end{gathered}
$$

at or near the throat.

At the angular horizons $\varphi_{2}=\varphi_{2}^{c}$ and $\varphi_{2}=2 \pi-\varphi_{2}^{c}$, we have $\rho c^{2}-\sigma=0$. All of these results have been obtained for the specific metric where $\Phi=0$, but it is easy to check that they are still valid for any metric component $g_{t t}$, provided it is everywhere finite. It follows that for an observer moving through the ringhole's throat with a sufficiently large speed, $\gamma>>1$, the energy density

$$
\gamma^{2}\left(\rho c^{2}-\sigma\right)+\sigma
$$

will be negative for $2 \pi-\varphi_{2}^{c}>\varphi_{2}>\varphi_{2}^{c}$ and positive for $2 \pi-\varphi_{2}^{c}>\varphi_{2}>\varphi_{2}^{c}$. 
(iv) From the above results one would expect lensing effects to occur at or near the throat: the mouths would act like a diverging lens for world lines along $2 \pi-\varphi_{2}^{c}>\varphi_{2}>\varphi_{2}^{c}$, and like a converging lens for world lines along $-\varphi_{2}^{c}<\varphi_{2}<\varphi_{2}^{c}$. Of course, no lensing action of the mouths could be expected at $\varphi_{2}=\varphi_{2}^{c}$ and $\varphi_{2}=2 \pi-\varphi_{2}^{c}$. Null-ray propagation reveals that focusing or defocusing of a bundle of rays is governed by the integral of the stress-energy tensor $[3,8]$. For the mouths to defocus rays, such an integral must be negative; i.e. $\int_{l_{1}}^{\infty} d l e^{-\Phi}\left(\rho c^{2}-\sigma\right)<0$, for any $l_{1}<0$, and positive if the mouths focus the rays. We shall perform this integration at the extreme cases $\varphi=\pi$ and $\varphi_{2}=0$. Using (3.11) and $\rho c^{2}-\sigma=\left(\frac{n}{r}\right)^{3}\left(T_{0}^{0}-T_{1}^{1}\right)$, we first get for $\varphi_{2}=\pi$ (for which $r=m=n=a+a)$

$$
\begin{gathered}
I_{\varphi_{2}=\pi}=\left.\frac{8 \pi G}{c^{4}} \int_{l_{1}}^{\infty} d l\left(\rho c^{2}-\sigma\right)\right|_{\varphi_{2}=\pi}= \\
{\left[-\frac{l}{l^{2}+b_{0}^{2}}-\frac{b_{0}}{a^{2}} \arccos \left(\frac{b_{0}}{\sqrt{l^{2}+b_{0}^{2}}}\right)\right.} \\
\left.+\frac{l}{a^{2}}-\frac{1}{a} \ln \left(2 l+2 \sqrt{l^{2}+b_{0}^{2}}\right)\right]\left.\right|_{l_{1}} ^{\infty} .
\end{gathered}
$$

The condition $a \geq b>l$ implies that we must make $a \rightarrow \infty$ while performing the integration limits, so the above expression reduces to $I_{\varphi_{2}=\pi}=-\frac{\left|l_{1}\right|}{l_{1}^{2}+b_{0}^{2}}<0$. At $\varphi_{2}=0$ (for which $r=m=-n=a-b$ ), we likewise obtain $I_{\varphi_{2}=0}=-I_{\varphi_{2}=\pi}>0$. Since at $\varphi_{2}=\varphi_{2}^{c}$ and $\varphi_{2}=2 \pi-\varphi_{2}^{c}, n=0$ and hence $I_{\varphi_{2}=\varphi_{2}^{c}}=0$, the above results can readily be generalized to:

$$
\begin{gathered}
\int_{l_{1}}^{\infty} d l e^{-\Phi}\left(\rho c^{2}-\sigma\right)<0 \text { for } 2 \pi-\varphi_{2}^{c}>\varphi_{2}>\varphi_{2}^{c} \\
\int_{l_{1}}^{\infty} d l e^{-\Phi}\left(\rho c^{2}-\sigma\right)>0 \text { for }-\varphi_{2}^{c}<\varphi_{2}<\varphi_{2}^{c}
\end{gathered}
$$

for any $\Phi$ which is everywhere finite.

Thus, as it was expected, the mouths act like a diverging lens for $2 \pi-\varphi_{2}^{c}>\varphi_{2}>\varphi_{2}^{c}$, and hence a bundle of light rays that enters one mouth originally converging to the direction tangent to the torus at points on circunference at any $2 \pi-\varphi_{2}^{c}>\varphi_{2}>\varphi_{2}^{c}$ must emerge from the same points of the other ringhole's mouth diverging from the same direction. Quite remarkably, unlike in spherical wormholes, the ringhole's mouths act also like a converging lens. This happens for $-\varphi_{2}^{c}<\varphi_{2}<\varphi_{2}^{c}$, where a bundle of light rays entering one mouth originally diverging emerges from the other mouth converging into the given direction.

\section{RINGHOLE TIME MACHINE}

The traversible ringhole space that we have considered in Sec. III can be regarded as a Misner space $[9,10]$ with the identified flat planes replaced for identified tori when we give these tori vanishing relative velocity $v=0$; i.e. it is equivalent to extract two tori with given geometric parameters $a, b$ from three-dimensional Euclidean space, and identify the torus surfaces, so when you enter the surface of the right torus, you find yourself emerging from the surface of the left torus, and vice versa. In Minkowski spacetime, the ringhole is then obtained identifying the two world conccentric tube pairs swept out by the two tori, with events at the same Lorentz time identified.

In order to convert a ringhole into time machine, one must set one of the ringhole toroidal mouths in motion at a given speed toward the other mouth, and identify then the two ringhole mouths to each other. Before discussing the effects that the toroidal shape of the ringhole mouths may cause in the pathologies of Misner and spherical wormhole spaces, let us derive the spacetime metric of such an accelerating ringhole. Assuming the right mouth to be the moving mouth, just outside the right asymptotic rest frame, the transformation of the ringhole coordinates into external, Lorentz coordinates with metric $d s^{2} \simeq-d T^{2}+d X^{2}+d Y^{2}+d Z^{2}$, can be written as

$$
\begin{gathered}
T=T_{R}+v \gamma l \sin \varphi_{2}, \quad Z=Z_{R}+\gamma l \sin \varphi_{2} \\
X=m_{l} \sin \varphi_{1}, \quad Y=m_{l} \cos \varphi_{1},
\end{gathered}
$$

where $v=\frac{d Z_{R}}{d T_{R}}$ is the velocity of the right mouth; $Z=$ $Z_{R}(t), T=T_{R}(t)$ is the world line of the right mouth's center with $d t^{2}=d T_{R}^{2}-d Z_{R}^{2}$, and $\gamma$ is the relativistic factor $\gamma=\left(1-v^{2}\right)^{-\frac{1}{2}}$. On the other hand, just outside the left asymptotic rest frame, the transformation is

$$
T=t, \quad Z=Z_{L}+l \sin \varphi_{2},
$$

with the expressions for $X$ and $Y$ the same as just for outside the right asymptotic rest frame. Here, $Z_{L}$ is the time-independent $Z$ location of the left mouth's center. Then the metric inside the accelerating ringhole and outside but near its mouths becomes

$$
d s^{2}=-\left[1+g l F(l) \sin \varphi_{2}\right]^{2} e^{2 \Phi} d t^{2}+d l^{2}+m_{l}^{2} d \varphi_{1}^{2}+b^{2} d \varphi_{2}^{2},
$$

where $g=\gamma^{2} \frac{d v}{d t}$ is the acceleration of the right mouth and $\Phi$ is the same function as for the original static ringhole. $F(l)$ is a form factor that vanishes in the left half of the ringhole, $l \leq 0$, and rises monotonously from 0 to 1 as one moves rightward from the throat to the right mouth [3]. In obtaining (4.1) we have also used $d v=g \frac{d t}{\gamma^{2}}$, $d t=\frac{d T_{R}}{\gamma}$ and $d \gamma=v g \gamma d t$. This metric is a generalization to toroidal symmetry of the metric used by Morris et 
al. for accelerating spherical wormholes [3]. Since the symmetry axis of the ringhole is taken again to be the axis $z$, reduction from (4.1) to the spherical wormhole metric can also be achieved by using the transformation $a \rightarrow 0, \varphi_{2} \rightarrow \theta+\frac{\pi}{2}, \varphi_{1} \rightarrow \phi$, as one should expect.

CTCs in accelerating ringhole space are originated at sufficiently late times by exactly the same causes as in Misner or accelerating spherical wormhole spaces [3,8]: on the left mouth the Lorentz time, $T$, and the proper time, $\tau$, coincide, $\tau=T$, but on the right mouth $\tau=\frac{T}{\gamma}$; this time dilation must ultimately lead to CTCs which would only appear after reaching a chronology (Cauchy) horizon. Such a horizon divides the full ringhole spacetime into a chronal region and a non-chronal region where CTCs appear. Like in Misner and accelerating spherical wormhole spaces, there will be two families of timelike geodesics in the chronal region: rightward geodesics and leftward geodesics, both possessing their own chronology horizons and non-chronal regions [11].

The mouth's lensing actions of the accelerating ringhole drastically change the geometry of the chronology horizon for any $\varphi_{2}$, except $\varphi_{2}=\varphi_{2}^{c}$ and $\varphi_{2}=2 \pi-\varphi_{2}^{c}$, at which cases the horizons remain flat, null surfaces, like in Misner space. In all the cases, one of the Cauchy horizons is no longer a flat, null surface for any $\varphi_{2} \neq \varphi_{2}^{c}$, $2 \pi-\varphi_{2}^{c}$. All that remains then is a compact fountain $[5,9]$ and, roughly speaking, a light cone at one of the ringhole's mouths [12]. If you go in through the ringhole along a world line where $2 \pi-\varphi_{2}^{c}>\varphi_{2}>\varphi_{2}^{c}$, the rightward chronology horizon and non-chronal region are destroyed, so then the accelerating ringhole appears to be only endowed with a leftward chronology horizon and non-chronal region. In this case, there will be, roughly speaking, a future light cone of point $P$ on the ringhole's left mouth (Fig. 3). The chronology horizon becomes then the boundary of the future Cauchy development of the fountain, $C_{R}$, generated by null geodesics which are past-directed, to asymptote and enter the compact fountain $C_{R}$. It can be said to be a compactly-generated horizon which is the location of onset of rightward CTCs [5]. Thus, for world lines with $2 \pi-\varphi_{2}^{c}>\varphi_{2}>\varphi_{2}^{c}$, everything happens like in accelerating spherical wormholes and, therefore, if you could use an accelerating ringhole to time travel along one such world lines, then you would find unphysical violation of the classical averaged null energy condition.

However, if you go in through the ringhole along a world line which passes by the region where $-\varphi_{2}^{c}<\varphi_{2}<$ $\varphi_{2}^{c}$ at or near the throat, then the throat would act like a converging lens for rightward propagation, and like a diverging lens for leftward propagation (which takes place backward in time due to time dilation of the accelerating right mouth), both with focal length $f \simeq \frac{b_{0}}{2}$. The classical instability caused by the pileup of leftwardpropagating waves on the leftward horizon can then be eliminated by particle-defocusing if large separations between mouths are used, so that $\frac{B b_{0}}{2 D}<<1$, where $B$ is the Doppler frequency-shifting factor and $D$ is the distance between ringhole mouths as measured along the fountain [3]. Therefore, the leftward chronology horizon and non-chronal region are destroyed and the accelerating ringhole becomes in this case endowed with only the rightward chronology horizon and non-chronal region.

For $-\varphi_{2}^{c}<\varphi_{2}<\varphi_{2}^{c}$ at or near the throat, the radical changes of the horizon geometry are then different of those occurring in the case of world lines passing always through the region where $2 \pi-\varphi_{2}^{c}>\varphi_{2}>\varphi_{2}^{c}$ (see Sec. $\mathrm{V})$. Now, roughly speaking, we have a past light cone of point $Q$ also on the ringhole's right mouth (Fig. 3). This past light cone lies in the past Cauchy development of the compact fountain, $C_{L}$, and has as boundary a chronology horizon compactly-generated by null geodesics which now are future-directed, and asymptote and enter the fountain $C_{L}$. Such a horizon is the location of onset of the chronal curves. All the CTCS are situated in the future of this Cauchy horizon.

The crucial difference with respect to Misner and spherical wormhole spaces is that in ringhole space, on world lines with $-\varphi_{2}^{c}<\varphi_{2}<\varphi_{2}^{c}$ at or near the throat, you can traverse CTCs without ever encountering a region with negative energy density. At or in the close neighborhood of the angular horizons, the energy density is always nonnegative and, since the ringhole spacetime is asymptotically flat, the focusing theorem [13] should then hold:

$$
\frac{d^{2} A_{K}^{\frac{1}{2}}(P)}{d \lambda_{K}^{2}} \leq 0
$$

where $A_{K}$ is the cross-sectional area generated by the intersection of the infinitesimal bundle $K$ of null-geodesic generators of the angular horizons with a spacelike slice at point $P$, and $\lambda_{K}$ is affine parameter along the bundle $K$. Furthermore, it follows from (3.11) that for $b>>$ $b_{0}$ the energy density turns out to become positive for $2 \pi-\varphi_{2}^{c}>\varphi_{2}>\varphi_{2}^{c}$, and negative for $-\varphi_{2}^{c}<\varphi_{2}<\varphi_{2}^{c}$, for sufficiently large $\gamma$. Then, after $b \simeq \sqrt{2} b_{0}$, the situation is inverted, the energy density of region $2 \pi-\varphi_{2}^{c}>\varphi_{2}>\varphi_{2}^{c}$ becoming negative and that of region $-\varphi_{2}^{c}<\varphi_{2}<\varphi_{2}^{c}$ becoming positive, at or near the throat, for large $\gamma$.

As one traverses the ringhole from one mouth to another (Fig. 4), if we restrict to the region before reaching the throat, the angular horizons do not have any curvature singularity which they can hit in the future and therefore

$$
\frac{d A_{K}^{\frac{1}{2}}(P)}{d \lambda_{K}} \geq 0 .
$$

So, like for event horizon of black holes, the total crosssectional area $A=\sum_{K} A_{K}$ of the ringhole angular horizon halves before the throat cannot decrease toward the future.

Any object with positive energy moving toward the throat through the region $2 \pi-\varphi_{2}^{c}>\varphi_{2}>\varphi_{2}^{c}$ (before 
reaching the cross-section at $\left.b \simeq \sqrt{2} b_{0}\right)$ will be increasingly pulled gravitationally out from that region, and then into the region $-\varphi_{2}^{c}<\varphi_{2}<\varphi_{2}^{c}$, across the angular horizons at around $\sqrt{2} b_{0}$, as the object approaches the throat (Fig. 4). One must choose then the region where $2 \pi-\varphi_{2}^{c}>\varphi_{2}>\varphi_{2}^{c}$, up to $b \simeq \sqrt{2} b_{0}$, before reaching the throat, as the causal past of the future null infinity, $J^{-}\left(I^{+}\right)$; that is the location of observers inside the ringhole that can send curves to future null infinity $I^{+}$. Then, the union of angular horizons at around $b \simeq \sqrt{2} b_{0}$, before reaching the throat, will be the future boundary $j^{-}\left(I^{+}\right)$ of $J^{-}\left(I^{+}\right)$for such observers. The null generators of the union of the angular horizons up to $b \simeq \sqrt{2} b_{0}$ can then be divided in infinitesimal bundles $K$ whose intersection with spacelike slices defines the cross-sectional areas $A_{K}$ satisfying (4.2), so as closed two-surfaces which are the angular horizons at the given times. For observers in $\jmath^{-}\left(I^{+}\right)$, the past singularity that must occur as $b \rightarrow a$ in region $-\varphi_{2}^{c}<\varphi_{2}<\varphi_{2}^{c}$ does not matter for the stability of the horizons, because bundles of null-geodesic generators of the angular horizons can always be inserted in the future of the past null infinity.

However, for observers traveling through the ringhole, in the region $-\varphi_{2}^{c}<\varphi_{2}<\varphi_{2}^{c}$, after the throat, there must also be a curvature singularity in the future infinity at $b \rightarrow a$ which the angular horizons will unavoidably hit. From the focusing theorem it follows then $[9,13]$

$$
\frac{d A_{K}^{\frac{1}{2}}(P)}{d \lambda_{K}} \leq 0 .
$$

Although the singularity that lies in the future infinity of region $-\varphi_{2}^{c}<\varphi_{2}<\varphi_{2}^{c}$ destroys the horizons, such a singularity would not be visible from the future null infinity $I^{+}$of the region $2 \pi-\varphi_{2}^{c}>\varphi_{2}>\varphi_{2}^{c}$, as it is on the same asymptotic spacelike slice. After reaching the throat, the object with positive energy will be gravitationally pulled out from region $-\varphi_{2}^{c}<\varphi_{2}<\varphi_{2}^{c}$, and into the region $2 \pi-\varphi_{2}^{c}>\varphi_{2}>\varphi_{2}^{c}$ again. From $b \simeq \sqrt{2} b_{0}$ onward, after the throat, one must choose then the region where $2 \pi-\varphi_{2}^{c}>\varphi_{2}>\varphi_{2}^{c}$ as the causal future of the past null infinity, $J^{+}\left(I^{-}\right)$; that is the location of observers inside the ringhole that can receive curves from the past null infinity $I^{-}$. In this case, for such observers the union of angular horizons at around $b \simeq \sqrt{2} b_{0}$, after the throat, will be the past boundary $j^{+}\left(I^{-}\right)$of $j^{+}\left(I^{-}\right)$, and, thereafter, their infinitesimal bundles of null-geodesic generators will, by intersecting spacelike slices, define cross-sectional areas $A_{k}$ satisfying (4.3), and closed two-surfaces that are the horizons at the given times.

We can then conclude that gravitational interaction inside the ringhole sets the kind of itineraries that can be followed when traversing a ringhole, without ever encountering a region with "exotic" matter (Fig. 4). If you go in through the ringhole moving mouth and come out through the stationary mouth by traversing a CTC along one such itineraries, you will go backward in time, without encountering yourself surrounded by matter that violates the averaged weak energy condition, or finding any classical instability. You can do similarly safe classical travels into the future if you go in through the stationary mouth and exit through the mouth that has moved, along these world lines.

\section{VACUUM FLUCTUATIONS}

Let us consider the effects of electromagnetic quantum vacuum polarization in accelerating ringholes. For this case, the point-splitting regularized Hadamard two-point function for a quantized, massless, conformally-coupled scalar field in the spacetime depicted in Fig. 3, for regions where the curvature vanishes [7], can be written in the form

$$
\begin{gathered}
G_{\text {reg }}^{(1) \pm}\left(x, x^{\prime}\right)= \\
\sum_{N=1}^{\infty} \frac{\xi}{4 \pi^{2} D}\left(\frac{b \xi}{2 D}\right)^{N-1}\left(\frac{1}{\lambda_{N}^{ \pm}\left(x, x^{\prime}\right)}+\frac{1}{\lambda_{N}^{ \pm}\left(x^{\prime}, x\right)}\right)
\end{gathered}
$$

where

$$
\xi=\left(\frac{1-v}{1+v}\right)^{\frac{1}{2}}<1,
$$

$D$ is the spatial length of a geodesic that connects points $x$ and $x^{\prime}$ by traversing the ringhole, and

$$
\lambda_{N}^{ \pm}\left(x, x^{\prime}\right)=\xi^{N} \frac{\sigma_{N}^{ \pm}}{\zeta_{N}}
$$

in which $\zeta_{N}=D\left(\frac{1-\xi^{N}}{1-\xi}\right)$ and $\sigma_{N}^{ \pm}$is the $N$ th geodetic interval between $x$ and $x^{\prime}$ for $(+) 2 \pi-\varphi_{2}^{c}>\varphi_{2}>\varphi_{2}^{c}$ and $(-)-\varphi_{2}^{c}<\varphi_{2}<\varphi_{2}^{c}$. $\lambda_{N}^{ \pm}$has been evaluated by the method of Hiscock and Konkowski used by Kim and Thorne [7]. In our case, by using Fig. 1, where the symmetry axis of the mouths is now taken to be $Y$, and the covering space [8] for the $Y-T$ plane of the ringhole spacetime, we can calculate the displacements at fixed times $T^{\prime}$ and $T$ of, respectively, copy 0 of $x^{\prime}$ and copy $N$ of $x$ from the covering-space throat location for $\varphi_{1}=0$, i.e.

$$
\begin{gathered}
\triangle \tilde{Y}_{0}^{ \pm}\left(x^{\prime}\right)=-(a \pm b)+m \\
\triangle \tilde{Y}_{N}^{ \pm}(x)=\xi^{-N}[(a \pm b)-m],
\end{gathered}
$$

and hence the corresponding geodetic intervals,

$$
\sigma_{N}^{ \pm}=\zeta_{N}\left[\left(\triangle \tilde{Y}_{N}^{ \pm} \xi^{N}-T\right) \xi^{-N}-\left(\triangle \tilde{Y}_{0}^{ \pm}-T^{\prime}\right)\right]
$$

when the points $x^{\prime}$ and $x$ are not on the symmetry axis, become 


$$
\begin{gathered}
\sigma_{N}^{ \pm}\left(x, x^{\prime}\right)= \\
\zeta_{N}\left\{\xi^{-N}\left[ \pm b\left(1 \pm \cos \varphi_{2}\right)-T\right]+\left[ \pm b\left(1 \pm \cos \varphi_{2}\right)+T^{\prime}\right]\right\} .
\end{gathered}
$$

If the points $x^{\prime}$ and $x$ are also slightly off the throat in the $Y$-direction, we finally have

$$
\begin{gathered}
\lambda_{N}^{ \pm}\left(x, x^{\prime}\right)= \\
\pm 2 b\left(1 \pm \cos \varphi_{2}\right)+Y-T-\left(Y^{\prime}-T^{\prime}\right) \xi^{N},
\end{gathered}
$$

where again the upper sign $(+)$ stands for $2 \pi-\varphi_{2}^{c}>\varphi_{2}>$ $\varphi_{2}^{c}$, and the lower sign (-) does for $-\varphi_{2}^{c}<\varphi_{2}<\varphi_{2}^{c}$, with $T=\tau, Y$ the Lorenzt coordinates of two-dimensional flat, Minkowski spacetime of the world line near the left mouth, and $T^{\prime}-\gamma(T-D)+v \gamma(Y-D), Y^{\prime}=\gamma(Y-$ $D)+v \gamma(T-D)$ the equivalent coordinates on a world line near the right mouth which is at rest at the origin.

According to Kim and Thorne [7], a $N$ th-polarized hypersurface, $H_{N}$, is formed by those events that join to themselves through closed null geodesics by traversing the ringhole $N$ times. On these hypersurfaces the quantum polarization of vacuum should diverge $[7,12]$. Then, upon collapsing the points $x^{\prime}$ and $x$ together, it turns out that there will be polarized hypersurfaces at times given by $\sigma_{N}^{ \pm}=0$; i.e.:

$$
T_{H_{N}}^{ \pm}= \pm\left(\frac{1+\xi^{N}}{1-\xi^{N}}\right) b\left(1 \pm \cos \varphi_{2}\right) .
$$

The chronology (Cauchy) horizons, $H^{ \pm}$, depicted in Fig. 3, are the limit as $N \rightarrow \infty$ of the times $T_{H_{N}}^{ \pm}$, and respectively nest the corresponding polarized hypersurfaces defined at times given by (5.4). On the symmetry axis, i.e. for $\varphi_{2}=\pi$ and $\varphi_{2}=0$, all polarized hypersurfaces $H_{N}$ occur at the same time only at $T=0$; away from the symmetry axis, one meets the $H_{N}$ one after another beginning with arbitrarily large $N$ and ending with $N=1$ as $T$ increases if $2 \pi-\varphi_{2}^{c}>\varphi_{2}>\varphi_{2}^{c}$, or as $T$ decreases if $-\varphi_{2}^{c}<\varphi_{2}<\varphi_{2}^{c}$. The nesting of the $H_{N}$ 's in the chronology horizon $\mathrm{H}^{+}$occurring at time

$$
T_{H^{+}}=+b\left(1+\cos \varphi_{2}\right)
$$

for $2 \pi-\varphi_{2}^{c}>\varphi_{2}>\varphi_{2}^{c}$ guarantees that, an observer entering the region of CTCs will pass first through the chronology horizon $\mathrm{H}^{+}$, and then successively through various $H_{N}$ 's. In the case $-\varphi_{2}^{c}<\varphi_{2}<\varphi_{2}^{c}$, the $H_{N}$ 's are located in the chronology horizon $H^{-}$occurring at time

$$
T_{H^{-}}=-b\left(1-\cos \varphi_{2}\right),
$$

so that the observer will first pass through successive $H_{N}$ 's and then enters the chronology horizon $H^{-}$. Perhaps in this case the observer could avoid experiencing the strong peaks of vacuum polarization by carefully choosing the moment at which she starts moving along a world line leading to CTCs.

Anyway, we see that semiclassical instabilities would also appear in the accelerating ringhole in both regions separately. Whether or not quantum-gravity effects or angular-horizon effects can cut off these divergences independently on each side of the angular horizon is a rather open question that has been the subject of much debate in recent years $[5,7]$.

We note nevertheless that the renormalized stressenergy tensor $T_{\mu \nu}^{ \pm}$, obtained by differentiating twice $G_{r e g}^{(1) \pm}$ [7], on polarized hypersurfaces with the same $N$ should satisfy $T_{\mu \nu}^{+}=-T_{\mu \nu}^{-}$, when the angle $\varphi_{2}$ in the interval $2 \pi-\varphi_{2}^{c}>\varphi_{2}>\varphi_{2}^{c}$ can be obtained from the corresponding angle in the interval $-\varphi_{2}^{c}<\varphi_{2}<\varphi_{2}^{c}$ by summing $\pi$ to the latter angle. From (5.4) we obtain in this case that the difference between the occurrence times of polarized hypersurfaces for the same $N$, nested in the two approximate light cones of Fig. 3 is given by

$$
\triangle T_{H_{N}}=T_{H_{N}}^{+}-T_{H_{N}}^{-}=2 b\left(\frac{1+\xi^{N}}{1-\xi^{N}}\right)\left(1+\cos \varphi_{2}\right),
$$

where $2 \pi-\varphi_{2}^{c}>\varphi_{2}>\varphi_{2}^{c}$. This precisely is the time the particles on the $N$ th-polarized hypersurface at a given $\varphi_{2}^{(i)}$ on the side of the angular horizon where $-\varphi_{2}^{c}<$ $\varphi_{2}<\varphi_{2}^{c}$ would take to go from their original location to that of the $N$ th-polarized hypersurface at $\varphi_{2}=\varphi_{2}^{(i)}+\pi$, on the other side of the angular horizon, reaching the latter hypersurface at exactly the time it is created.

It follows from the discussion in Sec. IV that, at or near the throat, everything having positive energy density, originally in the region $2 \pi-\varphi_{2}^{c}>\varphi_{2}>\varphi_{2}^{c}$, should cross the angular horizons into the region $-\varphi_{2}^{c}<\varphi_{2}<\varphi_{2}^{c}$, and everything having negative energy density, originally in the region where $-\varphi_{2}^{c}<\varphi_{2}<\varphi_{2}^{c}$, should cross the horizons in the opposite sense to get into the region $2 \pi-\varphi_{2}^{c}>\varphi_{2}>\varphi_{2}^{c}$. Moreover, since polarized hypersurfaces in region $2 \pi-\varphi_{2}^{c}>\varphi_{2}>\varphi_{2}^{c}$ occur later than those in region $-\varphi_{2}^{c}<\varphi_{2}<\varphi_{2}^{c}$, there will be then a flux of vacuum-polarized particles created by electromagnetic quantum fluctuations through the horizons, at or near the throat: that from the region where $-\varphi_{2}^{c}<\varphi_{2}<\varphi_{2}^{c}$ to the region where $2 \pi-\varphi_{2}^{c}>\varphi_{2}>\varphi_{2}^{c}$. Just once these particles have traveled an angular path $\triangle \varphi_{2}=\pi$ during a time $\triangle T_{H_{N}}$, crossing the angular horizons at $\varphi_{2} \simeq \frac{\pi}{2}$, they would reach the location where the otherwise identical vacuum-polarized particles with the opposite-sign energy are being created on polarized hypersurfaces with the same $N$, at exactly the time their counterparts arrive, so that, in the limit $\frac{b_{0}}{a} \rightarrow 0$, the quantum-vacuum polarizations occurring on both sides of the angular horizons would exactly cancel each other, leaving finally a vanishing polarized stress-energy tensor. 


\section{CONCLUSIONS}

In this paper we have explored the possibility that wormholes with the topology of a torus may exist and be used to construct a time machine. We call these wormholes ringholes. Ringholes with extremely large spacetime curvature could possibly be also spontaneously created in the quantum-gravity regime, and then pulled out from the quantum spacetime foam by a future technological process that would make them grow up to classical sizes [3]. The probability for the existence of such constructs in the foam is not known, but it has been recently established both from a theorem of Gannon [14] and by numerical computation [15] that, in the collapse of rotating collisionless matter, a toroidal horizon can be formed before the usual spherical horizon, provided we respect asymptotic flatness and the dominant energy condition.

By implicitly assuming the above or other possible mechanisms for ringhole creation, in this paper we have first considered the spacetime static metric corresponding to a ditribution of matter with the topology of a torus whose surface area and shape are kept constant. This solution shows two angular horizons on circunferences which are in between the maximum and minimum circunferences of the torus and parallel to them.

We then considered the static metric of traversible ringholes. These tunnels possess two distinct regions, separated by the angular horizons, which show different behaviours. On the region surrounding the maximum circunference, near the throat the ringhole acts like a diverging lens and is associated with an "exotic" stressenergy tensor having negative energy density. On the region surrounding the minimum circunference near the throat, the ringhole acts like a converging lens and associates with an ordinary stress-energy tensor allowing for positive energy density only.

Upon converting a ringhole into time machine by making one ringhole mouth to move with respect to the other and identifying the two mouths, it has been shown that there exists a chronology horizon on the inner region which opens up to a non-chronal region where time travels through closed timelike curves can be performed without ever encountering any violation of the averaged null energy condition. One could regard the angular horizons as implying some sort of censorship protecting the observers in the vecinity of the minimum circunference of the torus against experiencing the action of matter with negative energy density of the other angular horizon side, at or near the ringhole throat.

Polarized hypersurfaces with diverging stress-energy tensor have been shown to exist on both sides of the angular horizons: vacuum-polarized modes with positive energy are all located on the side of the maximum circunference, and those with negative energy only are on the other side of the angular horizons. Near the throat, the horizons have been found to bahave as one-directional membranes, so that all these modes would be expected to eventually end up all together confined in the region surrounding the maximum toroidal circunference, where they would exactly cancel each other in the limit of large tori with small throats, so that, in this case, the quantum vacuum polarization became unable to prevent ringholes and hence closed timelike curves to exist. Whether or not time travels through these ringholes would then entail an irretrievable loss of coherence [16] making the travels useless and very dangerous is a matter to be investigated.

\section{ACKNOWLEDGMENTS}

For helpful comments, the author thanks C. Sigenza and G. Mena Marugn. This research was supported by DGICYT under research projects N PB94-0107 and N PB930139.

[1] K.S. Thorne, Black Holes and Time Warps (W.W. Norton and company, New York, 1994).

[2] K. Gdel, Rev. Mod. Phys. 21, 447 (1949).

[3] M.S. Morris and K.S. Thorne, Am. J. Phys. 56, 395 (1988); M.S. Morris, K.S. Thorne and U. Yurtsever, Phys. Rev. Lett. 61, 1446 (1988); see also: G. Klinkhammer, Phys. Rev. D43, 2542 (1991); U. Yurtsever, Class. Quantm Grav. 7, L251 (1990); 8, 1127 (1991); M. Visser, Phys. Rev. D39, 3182 (1989); Nucl. Phys. B328, 203 (1989); V.P. Frolov and I.D. Novikov, Phys. Rev. D42, 1057 (1990); R. Wald and U. Yurtsever, Phys. Rev. D44, 403 (1991); F. Echeverria, G. Klinkhammer and K.S. Thorne, Phys. Rev. D44, 1077 (1991).

[4] J. Gott, Phys. Rev. Lett. 66,1126 (1991).

[5] S.W. Hawking, Phys. Rev. D46, 603 (1992).

[6] N.D. Birrell and P.C.W. Davies, Quantum Fields in Curved Space (Cambridge University Press, Cambridge, UK, 1982).

[7] W.A. Hiscock and D.A. Konkowski, Phys. Rev. D26, 1225 (1982); S.-W. Kim and K.S. Thorne, Phys. Rev. D43, 3929 (1991); K.S. Thorne, Ann. N.Y. Acad. Sci. 63, 182 (1991); V.P. Frolov, Phys. Rev. D43, 3878 (1991).

[8] K.S. Thorne, in: Directions in General Relativity. Proceedings of the 1993 International Symposium, Maryland; in honor of Charles Misner, Vol. 1, edited by B.L. Hu, M.P. Ryan Jr. and C.V. Vishveshwara (Cambridge University Press, Cambridge, UK, 1993).

[9] S.W.Hawking and G.F.R. Ellis, The Large Scale Structure of Space-Time (Cambridge University Press, Cambridge, UK, 1973).

[10] C.W. Misner, in: Relativity Theory and Astrophysics I. Relativity and Cosmology, edited J. Ehlers (American Mathematical Society, Providence, RI, 1967); C.W. Misner and A.H. Taub, Sov. Phys. JETP 28, 122 (1969).

[11] This can be rather shown if we take Minkowski spacetime as the covering space $[8,9]$ for ringhole space. This can be 
constructed by lining up a sequence of copies of ringhole space, side by side, each one boosted by speed $v$ relative to the last one.

[12] J. Friedman, M.S. Morris, I.D. Novikov, F. Echeverria, G. Klinkhammer, K.S. Throne and U. Yurtsever, Phys. Rev. D42, 1915 (1990).

[13] C.W. Misner, K.S. Thorne and J.A. Wheeler, Gravitation (Freeman and Co., San Francisco, 1970).

[14] D. Gannon, Gen. Relat. Grav. 7, 219 (1976).

[15] S.A. Hughes, C.R. Keeton, P. Walker, K. Walsh, S.L. Shapiro and S.A. Teukolsky, Phys. Rev. D49, 4004 (1994).

[16] S.W. Hawking, Phys. Rev. D52, 5681 (1995).

\section{Legend for Figures}

- Fig. 1. Cartesian coordinates on the two-dimensional torus. Any point $P$ on the torus surface can be labeled by parameters $a, b, \varphi_{1}, \varphi_{2}$.

- Fig. 2. Embedding diagram for a spacetime ringhole that connects two regions of the universe.

- Fig. 3. A spacetime ringhole with the right mouth moving toward the left. Also depicted are the fountains $(C)$ and the chronology horizons $\left(H^{ \pm}\right)$for the regions on both sides of the angular horizons at $\varphi_{2}=\varphi_{2}^{c}$ and $\varphi_{2}=2 \pi-\varphi_{2}^{c}$.

- Fig. 4. Half of a $\varphi_{1}=$ Const. section of a ringhole depicting the different regions inside it (see the text). The dashed line is an example of a trajectory that never passes through a region with negative energy density, even for large values of the relativistic factor $\gamma$. 
This figure "fig1-1.png" is available in "png" format from: http://arXiv.org/ps/gr-qc/9608059v1 
This figure "fig1-2.png" is available in "png" format from: http://arXiv.org/ps/gr-qc/9608059v1 
This figure "fig1-3.png" is available in "png" format from: http://arXiv.org/ps/gr-qc/9608059v1 
This figure "fig1-4.png" is available in "png" format from: http://arXiv.org/ps/gr-qc/9608059v1 